\title{
ETHNOGRAPHIE DU GECEKONDU. UN HABITAT AUTOCONSTRUIT DE LA PÉRIPHÉRIE URBAINE
}

Tahire Erman et traduction de Sylvie Muller

Presses Universitaires de France | « Ethnologie française »

2014/2 Vol. 44 | pages 267 à 278

ISSN 0046-2616

ISBN 9782130628927

Article disponible en ligne à l'adresse :

https://www.cairn.info/revue-ethnologie-francaise-2014-2-page-267.htm

Distribution électronique Cairn.info pour Presses Universitaires de France.

(C) Presses Universitaires de France. Tous droits réservés pour tous pays.

La reproduction ou représentation de cet article, notamment par photocopie, n'est autorisée que dans les limites des conditions générales d'utilisation du site ou, le cas échéant, des conditions générales de la licence souscrite par votre établissement. Toute autre reproduction ou représentation, en tout ou partie, sous quelque forme et de quelque manière que ce soit, est interdite sauf accord préalable et écrit de l'éditeur, en dehors des cas prévus par la législation en vigueur en France. Il est précisé que son stockage dans une base de données est également interdit. 


\title{
Ethnographie du gecekondu. Un habitat autoconstruit de la périphérie urbaine
}

Tahire Erman

Université de Bilkent

\author{
RÉSUMÉ \\ Tahire Erman \\ Siyaset Bilimi Bölümü, Bilkent Universitesi \\ Ankara 06800 \\ Turquie \\ tahire@bilkent.edu.tr
}

L'auteur donne la parole à des habitants de quartiers de gecekondu (bâtisseurs sans permis de leur propre maison), qui, dans le cadre de projets de transformation urbaine, ont été relogés dans les tours de cités construites par des promoteurs publics ou privés. Le récit de leur expérience à la fois vécue et remémorée révèle une valorisation des gecekondu par leurs anciens occupants, qui contraste fortement avec la dévalorisation dont ces constructions sont l'objet dans le discours dominant. Au cours de la transformation récente des quartiers de gecekondu en cités (HLM), leur valeur d'échange tend à éclipser leur valeur d'usage. Pourtant, nostalgiques de leur vie d'avant, les anciens habitants continuent de valoriser leur gecekondu.

Mots-clés: Cité. Gecekondu. Migrants ruraux/urbains pauvres. Récits. Turquie.

Cet article s'attache aux points de vue des classes urbaines inférieures, composées principalement de migrants ruraux, qui vivent à la périphérie des villes dans des maisons qu'ils ont eux-mêmes bâties sur des terrains ne leur appartenant pas. En Turquie, on appelle cet habitat informel gecekondu $u^{1}$, qui signifie littéralement "installé, ou dressé, dans la nuit ". Notre étude s'impose d'autant plus aujourd'hui que la suppression des gecekondu de la scène urbaine est en cours, et la population se trouve relogée dans des ensembles inadaptés à son ancien mode de vie. Les autorités justifient leur intervention en attribuant aux gecekondu la responsabilité des maux de la ville : de plus en plus stigmatisés, ils seraient les repaires du crime et du vice, occupés par des envahisseurs qui accaparent en toute illégalité les rares terres urbaines. Or la voix de leurs habitants est à peine entendue alors que, en quittant leur existence quasi villageoise pour les tours TOKI ${ }^{2}$ d'un projet de rénovation urbaine $\left(\mathrm{PTU}^{3}\right)$, ils affrontent un changement de vie aussi radical que brutal. Le but de cet article est d'y remédier en informant le lecteur sur le vécu et la perception de ces populations ${ }^{4}$.

\section{- La formation des gecekondu}

En Turquie, la migration de la campagne vers les grandes villes commence à la fin des années 1940, en réponse à la mécanisation de l'agriculture et à l'industrialisation urbaine qui accompagnent l'insertion dans le système capitaliste. Les migrants empruntent les axes routiers qui relient les marchés aux lieux de production agricole. En l'absence de logements accessibles à cette population pauvre, les gecekondu font leur apparition. En cette période de développementalisme national, l'État donne la priorité à l'industrialisation au détriment du logement social [Şenyapilı, 1982]. Aussi les migrants construisent-ils eux-mêmes leur maison là où ils trouvent des emplois, à proximité des centres-ville ou à la périphérie des villes près des usines. Les gecekondu sont alors des cabanes généralement construites sur un terrain public, parfois sur un terrain privé sans autorisation ni titre de propriété, ou sur des terres agricoles divisées en lots avec un titre de propriété collective (hisseli tapu) [Yönder, 1987]. La fin du parti politique unique ${ }^{5}$ qui a lieu justement à cette époque 
favorise l'arrivée du populisme. Pour obtenir des voix, les candidats promettent de légaliser les gecekondu et de leur apporter les infrastructures et les services de base. Mais ces promesses électorales sont ensuite oubliées. Toutefois, dans le cadre de relations clientélistes, certains migrants ont réussi à obtenir un titre de propriété du terrain auprès de leur municipalité. En 1966, l'État entreprend de résoudre le «problème des gecekondu " en votant une loi en trois volets, la loi $\mathrm{n}^{\circ} 775$, qui prévoit de rénover et de légaliser les gecekondu bien entretenus occupant un emplacement adéquat, de détruire ceux qui sont en mauvais état et mal situés, et d'empêcher à l'avenir ce type d'habitations. Mais seul le premier volet est mis en œuvre. Les municipalités contribuent à la rénovation de quartiers entiers de gecekondu, principalement en fournissant l'équipement nécessaire à la construction d'infrastructures, tandis que les résidents apportent bénévolement la main-d'œuvre pour construire collectivement des routes, creuser des tranchées pour les conduites d'eau et installer des poteaux électriques [Erman, 2011]. Se forment ainsi des quartiers qui ressemblent à des villages. Les maisons de plain-pied sont entourées de jardins plantés d'arbres fruitiers, où les habitants cultivent des légumes, élevant parfois aussi des volailles et des vaches, qui, outre les produits frais, leur apportent un revenu supplémentaire en cas de vente tout en réduisant les dépenses. Les maisons possèdent souvent un four extérieur, utilisé collectivement par les femmes. Il permet de réaliser des économies mais aussi de savourer le "pain du village ".

Les relations sociales reposent alors sur des relations de coopération et d'entraide. En réponse aux difficultés de la vie urbaine, plusieurs familles se regroupent: en tissant des liens de bon voisinage, elles mettent en place des services de soins aux enfants, aux personnes âgées et aux malades. La construction des gecekondu se faisant généralement de façon collective par des migrants originaires du même endroit [Keyder, 2000], des groupes de voisinage « de type familial " se forment d'autant plus facilement. Enfin, le contrôle informel des "étrangers ", aisément repérés et interrogés, empêche le développement de la criminalité dans cet "espace défendable " (defensible space), selon l'expression d'Oscar Newman [1972]. L'Etat ne joue ainsi qu'un rôle mineur dans la création des communautés de migrants ruraux urbains qui peuvent reproduire librement leurs habitudes et leurs préférences tout en subissant l'influence de la ville. Marqués des stigmates de la "ruralité ", les gecekondu sont considérés comme des enclaves paysannes où vit une population ignare et inculte [Erman, 2001] ${ }^{6}$. Une tare rendue supportable par une vie entre soi à l'écart des citadins modernes.

Au fil des ans, le caractère autoconstruit et l'apparence villageoise des quartiers de gecekondu évoluent, ainsi que leur population. De nouveaux venus se mêlent aux résidents plus anciens, et les enfants des migrants eux-mêmes, socialisés en ville, côtoient les petits citadins des classes populaires. De plus, dans les années 1970, les terrains de gecekondu sont eux aussi soumis à la spéculation, sur le marché informel de l'immobilier, et on commence à y construire des immeubles d'habitation. Les classes moyenne et supérieure se détournant de plus en plus des centres urbains pollués et surpeuplés, les villes s'étendent et les terrains périphériques prennent de la valeur. Des groupes de type mafieux occupent de grandes surfaces de terre qu'ils vendent ensuite par lots aux personnes désireuses de construire un gecekondu. Les gains spéculatifs étant plus élevés à Istanbul, des immeubles de plusieurs étages se construisent eux aussi sans titre de propriété (les apartkondu). Le développement des zones périurbaines auquel ont participé plusieurs acteurs, allant du simple migrant au mafieux, s'est ainsi accompli en dehors de l'État, qui brille par son absence.

\section{- La transformation récente des gecekondu}

Dans les années 1980, lorsque la politique d'industrialisation par substitution aux importations cède le pas à une économie tournée vers l'exportation, l'État commence à s'intéresser aux gecekondu. Parallèlement, la libéralisation de l'économie et, par conséquent, l'ouverture du pays à la compétition internationale, mettent fin à la protection qu'il accordait jusque-là au secteur privé. Les nouveaux moteurs économiques sont l'exportation (dominée par l'industrie textile) et le tourisme, bientôt suivis par le bâtiment. La possibilité de générer des profits en transformant les périphéries urbaines ouvre une ère nouvelle pour cet habitat. Dans les années 1980, le gouvernement légifere afin de réorganiser le marché immobilier, votant notamment cinq lois d'amnistie entre 1983 et 1988. En légalisant les gecekondu et en mettant les terrains qu'ils occupent sur le marché officiel, il espère favoriser un boom de la construction. Dans les zones non couvertes par un plan d'aménagement urbain, des titres de propriété 
provisoires sont vendus aux occupants, qui seront remplacés par des titres définitifs après la mise en œuvre du plan. Dans les zones couvertes, ces titres de propriété donnent le droit de construire des bâtiments de quatre étages au plus. La transformation des gecekondu en immeubles d'habitation est l'œuvre de petits promoteurs qui achètent le terrain du propriétaire en échange de plusieurs appartements dans l'immeuble qu'ils vont y construire. Les emplacements choisis sont bien situés, à proximité de quartiers aisés, des principaux axes de transport ou sur des terrains plats pour un moindre coût, les collines rocheuses et escarpées ou la trop grande banlieue étant exclues. Il en résulte une transformation orientée des quartiers de gecekondu.

Les années 2000 marquent une nouvelle ère. Notamment sous la houlette du gouvernement néoconservateur et libéral de l'AKP, l'État commence à intervenir dans ces quartiers afin d'augmenter la rentabilité foncière. Ce sont généralement les municipalités qui s'en chargent dans le cadre de "Projets de transformation urbaine" (PTU). Les maires présentent leur mission comme un grand nettoyage de leur ville [Karaman, 2008]. Pour le premier ministre, Tayyip Erdoğan, les gecekondu sont des tumeurs qu'il faut retirer grâce aux PTU chargés de mener à bien cette opération chirurgicale [Ünsal et Kuyucu, 2010]. Aujourd'hui, de plus en plus de municipalités réalisent leurs PTU avec le concours de la TOKI, en intervenant dans les zones de gecekondu, pour les démolir le cas échéant. Obligés de quitter leur maison et leur quartier, de renoncer à un mode de vie familier et de s'adapter à un nouvel environnement, les habitants vivent l'expérience du déplacement. De vastes et luxueux lotissements construits par de gros promoteurs prennent la place des gecekondu détruits. Dans certains milieux, l'action de la токI est très critiquée et qualifiée de «transfert de propriété réalisé par l'Etat » [ibid.].

D'après le discours dominant, les PTU servent les intérêts des occupants qui, débarrassés de leur maison " hideuse ", " insalubre " et "délabrée ", sont installés dans un logement "moderne " et tout confort, avec des services haut de gamme. Dans la pratique, les habitants qui sont légalement propriétaires du terrain de leur gecekondu recevront un appartement dans la cité construite à la place par la TOKI, tandis que les autres, en contractant un crédit, seront relogés dans une tour de l'une des " cités sociales " ${ }^{7}$, généralement excentrées, de la TOKI. Les autorités reprochent aux habitants des gecekondu de ne pas apprécier les efforts déployés pour améliorer leur habitat. Des recherches récentes montrent toutefois que ces " cités modernes ", qualifiées de "prisons urbaines" par Ayfer Bartu-Candan et Biray Kolluoğlu [2008], posent de graves problèmes, alliant de nouvelles formes de pauvreté à l'exclusion sociale.

\section{—e relogement en cités HLM ou privées}

La cité de Karacaören-TOKI ${ }^{8}$ a été construite pour reloger les habitants dont les maisons ont été détruites dans le cadre du Projet de transformation urbaine d'Ankara-Nord' et qui, propriétaires de leur maison, n'avaient pas acquis la propriété de leur terrain. Ces propriétaires "illégaux ", selon la TOKI, peuvent ainsi posséder un appartement à Karacaören-TOKI en payant les traites d'un crédit pendant quinze ans. La cité de Bezirganbahçe-ToKI à Istanbul ${ }^{10}$ a elle aussi été construite pour les bâtisseurs sans titre de propriété dont les maisons ont été détruites dans le cadre du PTU d'Ayazma-Tepeüstü. Les conditions d'acquisition sont les mêmes à une différence près : la résistance collective des habitants d'Ayazma leur a permis d'obtenir du premier ministre une réduction de l'augmentation bisannuelle des versements mensuels. Dans le cadre du PTU de Şentepe, les gecekondu ont été démolis lorsque les propriétaires des terrains correspondant sur le plan d'aménagement à celui des tours d'habitation ${ }^{11}$ se sont entendus pour engager un promoteur privé, et céder leur parcelle contre un ou plusieurs appartements.

\section{Les cités de Karacaören-TOKI et de Bezirganbahçe-TOKI}

Ces deux cités construites par l'administration du logement social (TOKI) sont dotées des équipements habituels : un centre commercial, une école, un dispensaire et une mosquée, mais aussi plusieurs terrains de jeux et des bancs répartis dans la cité. Karacaören-TOKI comprend
34 immeubles de 12 étages, 4 de 15 étages et 44 de 4 étages. Selon un représentant de la TOKI, ces derniers ont été ajoutés au projet pour rompre la monotonie de l'ensemble. En revanche, les 55 tours de Bezirganbahçe-TOKI possèdent toutes 12 étages. 


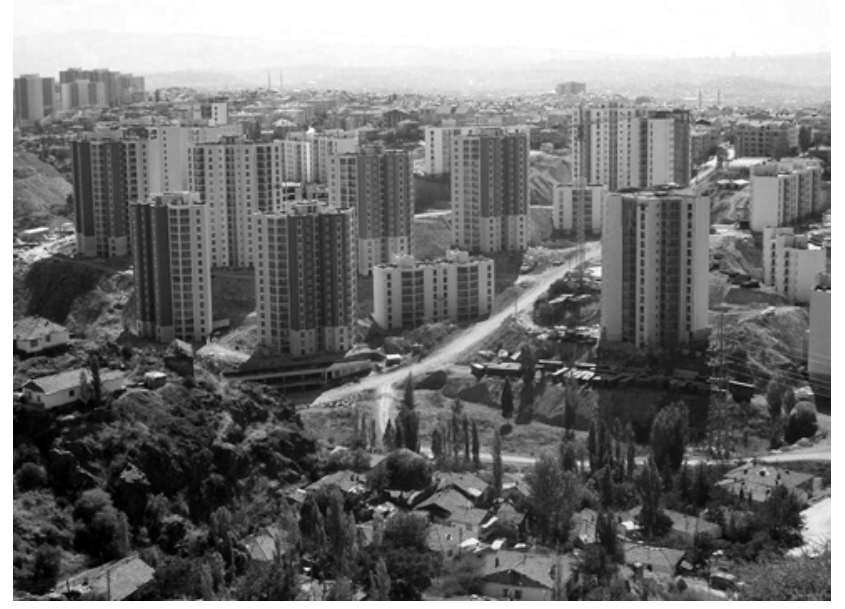

Photo 1: Tour d'habitation empiétant sur une zone de gecekondu, dans le nord d'Ankara. Photo de l'auteur, 2013.

Or, pour reprendre les termes utilisés par le chauffeur qui nous conduit sur le terrain, le style très moderne de ces cités offre un contraste "bizarre" avec leurs occupants, qui vaquent à des occupations "champêtres" vêtus "comme des paysans". Les femmes se rassemblent souvent au pied des immeubles ou dans les jardins attenants, assises dans l'ombre des tours. Hommes et femmes travaillent dans ces jardins, se disputant avec les gérants pour savoir si, outre les plantes et les arbres jugés acceptables pour une cité "moderne", ils peuvent semer des légumes. On voit souvent les femmes en train de laver des tapis, d'aérer des matelas ou même la laine des matelas, à proximité des immeubles dans l'espace public, en particulier dans la cité de Karacaören-TOKi. De plus, les chaussures et parfois les vélos sont fréquemment laissés devant la porte des appartements. La raison invoquée est la petitesse des logements, mais aussi la tradition et l'habitude. Des rappels au règlement intérieur sont régulière- ment placardés dans le hall d'entrée par le gérant de l'immeuble ou par un résident responsable.

On observe le même type de phénomène dans les centres commerciaux des cités. Les boutiques empiètent sur le passage et l'endroit parait bondé. Les commerces qui ferment sont vite remplacés et semblent se succéder dans un flux constant. Ces centres commerciaux de conception moderne, construits avec des matériaux nouveaux, dotés d'escalators et de panneaux rappelant tout ce qu'il est interdit de faire dans un lieu public, peuvent être très intimidants; les habitants des cités tentent néanmoins de se les approprier en les transformant, mais au prix d'incessants conflits avec les responsables du centre.

On peut ainsi observer une opposition marquée entre la conception "moderne " de la cité et son utilisation "rurale" par les habitants venus des gecekondu. Cette opposition crée des désaccords continuels sur la manière de se comporter dans les espaces communs, les pratiques de la vie quotidienne transgressant presque toutes le règlement établi par la TOKI.

À Başyazıc1-Çiğgem, l'idée de regrouper les anciens habitants de gecekondu dans deux tours séparées (sur les cinq que compte la cité) revient au promoteur. S'agissant d'un ensemble relativement petit, la cité n'a qu'une seule aire de jeu, les autres équipements se trouvant à proximité mais à l'extérieur. Comparée aux cités TOKI, l'application du règlement intérieur y est beaucoup plus stricte. Le gérant élu par les résidents nous a raconté qu'il avait ramassé toutes les chaussures devant les portes pour bien faire comprendre que cette pratique ne serait pas tolérée. Les femmes parlent entre elles lorsqu'elles surveillent leurs enfants qui jouent dehors, mais ne forment pas de petits groupes assis près des tours. Un agent de sécurité est posté à l'entrée de la cité, une femme en l'occurrence, qui a d'ailleurs refusé de laisser passer l'anthropologue sans l'autorisation expresse du comité de direction. En revanche, l'entrée de Karacaören-токі n'est pas gardée, et ses résidents

\section{Les cités privées du Projet de transformation urbaine de Şentepe}

Les cités du PTU du district ankariote de Şentepe sont beaucoup plus petites comparées aux cités de la TOKI. Les gecekondu sont progressivement remplacés par des tours d'habitation, le maire ayant fait de Sentepe un PTU et confié sa réalisation à des promoteurs privés. L'endroit ressemble aujourd'hui à un immense chantier. Başyazıcı-Çiğdem, le plus grand ensemble immobilier de Şentepe, se compose de 5 tours de 14 étages; un autre en compte 3 de 12 étages, et le reste une ou deux tours de plus de 10 étages. À Başyazıc1-Çiğdem, deux des tours étaient réservées aux propriétaires fonciers de gecekondu, les autres étant vendues sur le marché privé. 
sont souvent inquiets pour leur sécurité. Le portail de Bezirganbahçe-TOKI est surveillé, mais nous sommes entrés sans difficulté, et notre présence est passée inaperçue jusqu'au dernier jour : on nous a alors demandé d'obtenir une autorisation pour notre enquête auprès du bureau de la sécurité.

Ainsi, à Başyazıc1-Çiğdem, la soumission des habitants issus des gecekondu aux décisions des autres résidents, qui l'emportent en nombre, réduit la visibilité des activités "rurales » dans les espaces communs. Le règlement intérieur y est moins souple que dans les cités TOKI. Il importe en effet que la cité ne soit pas identifiée à un quartier de gecekondu, sous peine de voir chuter sa cote sur le marché de l'immobilier.

\section{- Deux quartiers de gecekondu}

Au début des années 1990, le quartier de Çukurca était couvert de gecekondu, avant de devenir un secteur urbain de la classe moyenne. Construit à flanc de colline, il faisait face aux immeubles d'un quartier de la classe moyenne supérieure située de l'autre côté de la vallée. De plain-pied ou d'un étage, les maisons étaient généralement entourées d'un jardinet. Éparpillées, elles étaient reliées entre elles par des chemins de terre et des dindons picoraient alentour : on se serait cru à la campagne. Le quartier de gecekondu de Nato Yolu dans le district ankariote de Mamak était quant à lui bien ordonné, des étudiants ayant tracé le plan de ce «territoire libéré » par la gauche dans les années $1970^{12}$. Les maisons étaient ici aussi de plain-pied ou d'un étage avec jardin. Situés à 10 kilomètres du centre-ville, ces gecekondu n'avoisinaient pas les quartiers des classes moyennes et supérieures, à la différence de ceux de Çukurca. Remplacés par des tours d'habitation, ils ont aujourd'hui presque disparu de l'avenue principale, mais se maintiennent à l'intérieur du quartier.

\section{- Le point de vue de la population}

"Le gecekondu (tel qu'il est) vécu ", "le gecekondu remémoré " et le "gecekondu raconté "sont les trois piliers qui soutiennent le point de vue des résidents sur leur habitat et leur mode de vie. En premier lieu, les entretiens de la première enquête menée dans le quartier ankariote de Çukurca permettent de comprendre le récit du "gecekondu vécu » au quotidien. Puis, les échanges avec les populations déplacées des cités TOKI ankariote de Karacaören et stambouliote de Bezirganbahçe éclairent le récit du "gecekondu remémoré ». Les entretiens avec les habitants des cités privés du PTU de Şentepe seront également utilisés à des fins comparatives, pour voir si vivre dans un gecekondu prend un sens différent selon que le déplacement est forcé ou volontaire. On ne peut bien comprendre ces récits sans les replacer dans l'expérience vécue par cette population, récemment relogée dans des tours d'habitation ${ }^{13}$. Enfin, les réponses des participants aux enquêtes plus récentes permettent d'élaborer le récit du "gecekondu raconté ". Pour terminer, le "gecekondu politisé " est abordé en prenant appui sur le quartier de Mamak-Nato Yolu : nous essayerons de comprendre les effets de la politisation récente du gecekondu sur la façon dont ses habitants se représentent la vie en son sein.

\section{- Le gecekondu vécu}

De la première enquête menée dans le quartier de Çukurca, il ressort que, selon les personnes, la préférence va au gecekondu ou à l'appartement. Les ménagères, les nouveaux venus en ville et les personnes âgées, en particulier, apprécient l'esprit communautaire, alliant prévenance et partage, qui règne dans les quartiers de gecekondu. [Erman, 1997]. Autres points forts cités par de nombreux participants : la liberté de faire ce qu'on veut chez soi et la possibilité de faire du bruit sans déranger ses voisins. Les espaces mi-privés, mi-publics autour des habitations, où les femmes se retrouvent, et bien sûr les jardins, sont également plébiscités. Certaines personnes se disent aussi attachées à la maison qu'elles ont tant peiné à bâtir pour en faire un endroit agréable à vivre.

Toutefois, pour les habitants de Çukurca, le gece$k o n d u$ présente des inconvénients. Il souffre notamment d'une qualité de construction médiocre, qui le rend non seulement difficile à nettoyer et à chauffer, mais nuisible à la santé en cas d'infiltrations. De nombreux résidents se plaignent également du manque d'infrastructures, des routes mal entretenues ou des eaux usées qui s'écoulent à l'air libre.

Ainsi, la liberté d'action, les bonnes relations de voisinage et la proximité de la nature s'accompagnent de désagréments importants: il faut se colleter avec des routes poussiéreuses ou boueuses selon la saison, des maisons mal chauffées, envahies par la poussière et l'humidité, dont les canalisations gèlent en hiver et le toit fuit. Enfin, la présence proche d'un quartier de la classe moyenne supérieure a sans doute stimulé l'envie de vivre dans un appartement. 


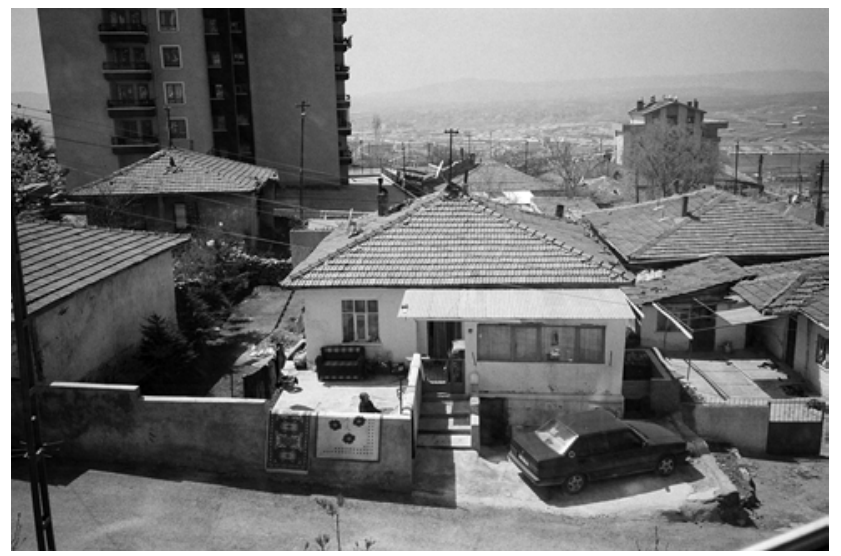

Photo 2 : L'utilisation de l'espace en plein air dans un gecekondu à Şentepe. Photo de l'auteur, 2013.

\section{- Le gecekondu remémoré}

- Les résidents de cités TOKI

Les habitants des cités font souvent de la vie dans les gecekondu un récit empreint d'une grande nostalgie. Ils évoquent cette époque avec regrets en exprimant des sentiments très forts. Or, leur nouveau lieu de résidence joue également un rôle dans cette remémoration.

Dans leur souvenir, ils assimilent le gecekondu occupé par une seule famille à un espace de liberté où, seuls maitres à bord, ils étaient chez eux. Voici un témoignage : «Dans un geckondu, tu vis librement, sans contrainte et tu es plus heureux; tu peux crier, chanter si tu veux, taper du pied et faire du bruit. Tu fais un barbecue dans ton jardin. Ici, on est comme dans un carcan, on doit sans cesse dire à nos enfants "arrête", "ne fais pas ci, ne laisse pas tomber ça", "marche sans faire de bruit" ". Pour respecter le règlement intérieur de la токI et éviter les plaintes des voisins, il faut ainsi brider son comportement et celui de ses enfants, dans les parties communes et jusque dans son propre appartement.

Aucun espace extérieur n'est prévu dans les cités de la тOKI pour accueillir les activités pratiquées dans les gecekondu (cuire le pain, laver les tapis, etc.), et permettre aux femmes de se retrouver ou aux familles de se réunir pour un mariage. Ces activités sont interdites, en particulier lorsque la direction de la TOKI s'occupe seule de la gérance. Or cela pose un problème car, par habitude autant que par nécessité économique, les résidents ne peuvent y renoncer. Ils n'ont alors pas le choix et cuisent le pain dans la cave, mettent les tapis à sécher sur le trottoir, étalent la laine des matelas sur les voitures en stationnement, tandis que les femmes s'assoient par terre devant les tours. Se forme ainsi, selon l'expression de notre chauffeur, un " environnement étranger ", qui n'est ni un village ni une ville. Témoignant d'un manque de culture et d'éducation urbaines chez les anciens habitants de gecekondu, ces comportements marquent les cités sociales de la тоKI d'un stigmate supplémentaire.

Conscient de ce stigmate, certains résidents tentent d'imposer les "règles de la vie collective " à ces voisins indisciplinés, créant des situations conflictuelles. En emménageant dans un appartement, les habitants de gecekondu deviennent particulièrement susceptibles, et peuvent réagir très violemment si quelqu'un leur dit comment ils doivent se comporter. La tolérance et la coopération en vigueur dans les gecekondu semblent avoir disparu, au moins dans les premiers temps ${ }^{14}$. Conflits et tensions s'installent également lorsque le gérant tente d'intervenir. Certains occupants, par exemple, persistent à laisser leurs chaussures devant la porte, par tradition, mais aussi, disent-ils, parce qu'elles sentent mauvais et que la place manque dans l'appartement. Or, pour le gardien et certains résidents, cette pratique constitue une pollution visuelle et une marque de ruralité.

En second lieu, la forte sociabilité vécue dans les quartiers de gecekondu, mais absente des tours d'habitation surpeuplées, alimente une profonde nostalgie. Dans le discours des personnes interrogées, les voisins se connaissaient et prenaient le temps de s'entraider dans ces quartiers, en mettant en commun leurs ressources, et en apportant un soutien psychologique ou financier à quiconque était malade ou dans le besoin. Inséparables, les femmes se recevaient à tour de rôle les unes chez les autres ou dans leur jardin respectif pour le petit déjeuner. Les hommes aussi étaient proches et se retrouvaient après le travail pour boire du thé et parfois jouer aux cartes. Ces petits groupes de voisins vivant à proximité fonctionnaient comme une famille élargie : hommes et femmes se comportaient en frères et sœurs, la ségrégation de genre ne s'appliquant pas, même dans les familles les plus conservatrices sur le plan religieux. Une femme se souvient : "C'était comme une famille, comme les membres d'une même famille. On se faisait confiance. On se retrouvait librement, on mangeait ensemble, on faisait des pique-niques ensemble. Nos enfants ont grandi ensemble, les enfants de nos voisins étaient pour nous comme nos enfants". Bien sûr, la vie dans les gecekondu n'était pas exempte de disputes ni de problèmes, mais " cela ne durait pas ». Elle s'accompagnait également de commérages et d'ingérences dans la vie privée, mais " rien de bien grave ". 
Un grand nombre de résidents se souviennent de la joie qu'ils éprouvaient au contact de la nature. Ils cultivaient leur jardin, y faisaient pousser des légumes, des fleurs et des arbres fruitiers. Ils prenaient plaisir à ce travail, à cueillir les fruits, à se reposer à l'ombre des arbres, ou simplement à écouter le chant des oiseaux. Une femme raconte : "L'été, je sortais dès que j'étais levée, je travaillais dans mon jardin, j'arrosais les plantes. J'en cueillais les fruits pour les manger. Chaque jour, il y avait un oiseau qui chantait joyeusement. J'adorais jardiner ». Et peu importe si, souvent, ce jardin n'était pas bien grand : en les remettant au contact de la terre, il leur permettait de se détendre.

La plupart des résidents regrettent l'absence de ces jardins dans les cités TOKI, sauf un petit groupe porté à se distancier de leurs voisins. Certains d'entre eux ont planté des fleurs et même des arbres devant et derrière leur tour. Mais d'autres ont essayé de faire pousser des légumes, avec le même résultat qu'à Bezirganbahçe-TOKI : à la consternation générale, ils ont tous été rasés par la tondeuse du jardinier de la TOKI. Si les fleurs évoquent l'urbanité, l'association des légumes avec le monde rural rend leur présence inacceptable.

Un autre aspect positif de la vie dans les gecekondu, au sein de petits groupes, est le sentiment de sécurité. Pendant la journée notamment, "on laissait la porte ouverte pour pouvoir aller et venir librement entre voisins "; " on ne fermait pas la porte à clé : un étranger sera toujours repéré par l'un ou l'autre d'entre nous ". En revanche, dans les tours des cités TOKI comptant chacune douze étages et quatre appartements par étage - cette maitrise de l'environnement ne peut plus s'exercer. Il est impossible de recréer dans ce nouveau milieu, surpeuplé et hétérogène, le même type de communauté : les vols se multiplient et la sécurité se dégrade. Tant et si bien que les résidents réclament des caméras de surveillance et du personnel qualifié pour protéger l'entrée de la cité.

Plus important encore, les gecekondu offraient un abri contre la compétitivité urbaine. On y menait une vie simple, sans se sentir obligé de se mettre en scène, en portant certains vêtements par exemple. En appartement, ce type de pressions commence à s'exercer. Cela concerne davantage les anciens habitants de gecekondu qui ont réalisé un profit en se relogeant dans le PTU de Şentepe ; ceux qui, relogés de force dans les cités TOKI, se sont endettés étant moins concernés. Dans les deux groupes, un grand nombre de familles ont acheté des meubles à crédit, et ceux qui n'en ont pas les moyens se sentent défavorisés.
Les problèmes inhérents aux gecekondu - chauffage, nettoyage, réparations - sont parfois mentionnés, notamment par un petit groupe de femmes qui rêvent d'ascension sociale. Cette évaluation globalement très positive peut s'expliquer ainsi : les habitants des tours TOKI ressentent plus fortement ce qui leur manque de leur ancienne vie que les difficultés qu'ils y rencontraient. Cela est particulièrement vrai des résidents qui définissent encore leur identité par rapport à leur quartier d'origine. Mais une autre explication est possible : ils ont quitté leur gecekondu contre leur gré et, outre le prêt qu'ils peinent déjà à rembourser, ils doivent faire face à de nouvelles dépenses, notamment les charges de copropriété. C'est le problème le plus souvent mentionné par presque toutes les personnes interrogées : les mensualités qu'ils devront payer pendant quinze ans pour devenir propriétaires de leur appartement et qui, en plus, augmentent deux fois l'an (en même temps que les salaires des fonctionnaires sur lesquels elles sont indexées). Voilà ce qui les rend nostalgiques de l'existence "insouciante " qu'ils menaient dans leur ancien quartier. Les conséquences économiques de leur changement de résidence affectent profondément leur nouvelle vie. Par exemple, ils disposent à présent du gaz de ville, mais s'ils n'arrivent pas à payer la facture ils se retrouvent sans chauffage. Les avantages de ce nouveau logement deviennent alors très relatifs. Enfin, s'ils ne peuvent plus payer les mensualités, ils perdront leur appartement.

Le quartier de gecekondu d'Ayazma, qui fut démoli dans le cadre du PTU de la municipalité de Küçükçekmece, occupe une place particulière dans la mémoire des résidents kurdes de la cité de Bezirganbahçe-TOKI : ils y étaient chez eux, entre proches, sans ingérence extérieure. Un jeune en garde un souvenir très fort :

On vivait bien là-bas. On avait la paix, on n'avait peur de rien, la police ne pouvait pas entrer. Mais ici tu n'as jamais la paix et tu as peur de tout, il n'y a ni fraternité ni amitié ; tu es toujours sur tes gardes, ils te collent facilement une étiquette. Là-bas, on se connaissait tous alors qu'ici tu ne vas pas chez ton voisin même quand tu n'as rien à lui demander. À Ayazma, tous les voisins se retrouvaient pour bavarder, moi je faisais du vélo, alors qu'ici ils nous laissent même pas nous asseoir dans un coin, il n'y a pas de place pour nous.

Et l'ami de ce jeune kurde d'ajouter : « Ayazma nous faisait du bien ; ici on se désintègre ".Voyons à présent si les personnes qui ont échangé le terrain de leur gecekondu contre un appartement, en négociant avec des promoteurs privés, tiennent un discours différent de celui des résidents des cités TOKI. 


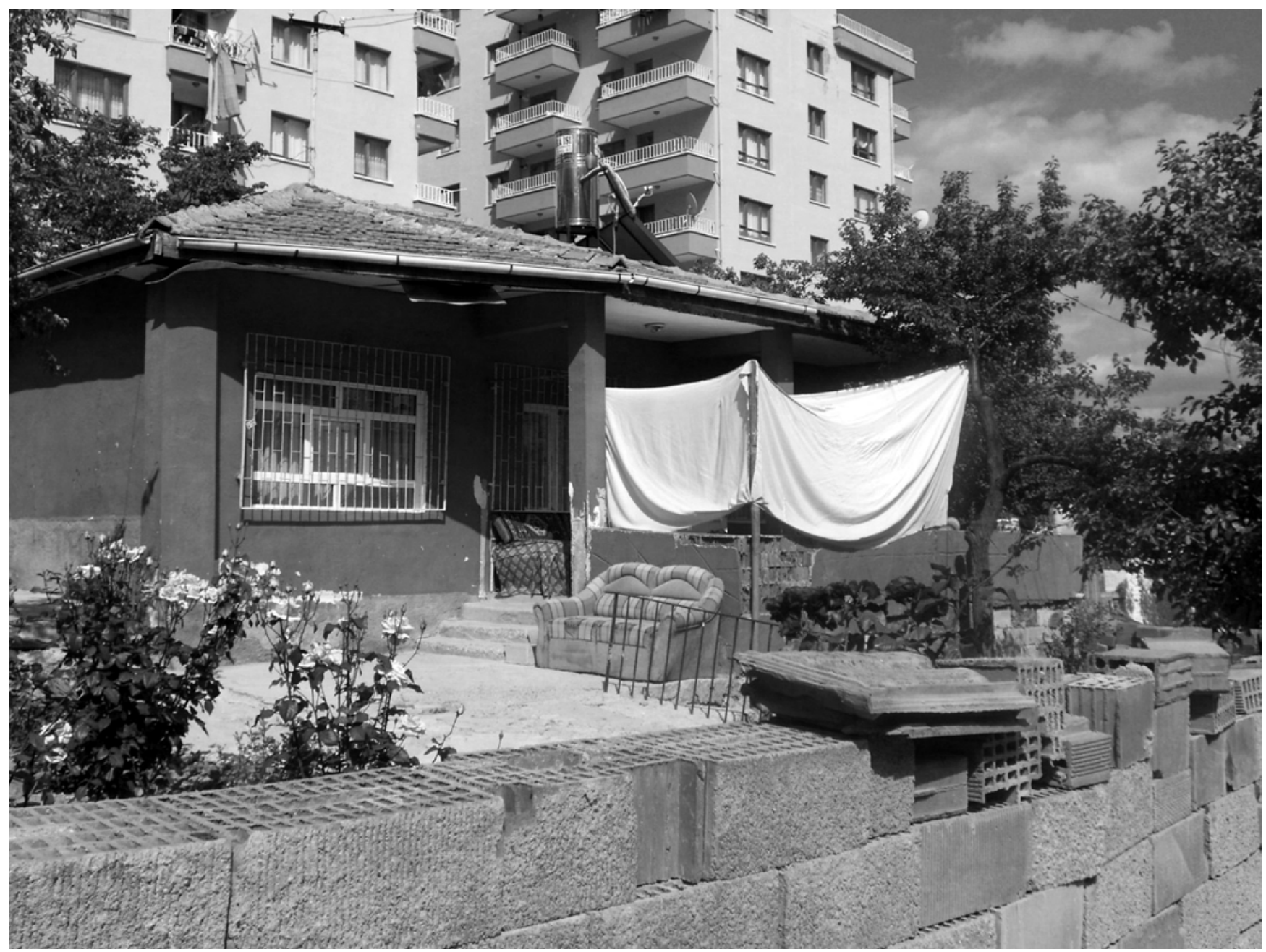

Photo 3 : Un gecekondu restant dans le milieu d'immeubles d'habitation à Şentepe. Photo de l'auteur, 2013.

- Les résidents des cités privées du PTU de Şentepe Les propriétaires fonciers de gecekondu ont obtenu à Şentepe des appartements de grande taille correspondant à leur demande : "Nous avons deux salles de bain, deux W.-C., trois chambres. On a toute la place qu'on veut. " Ils sont également satisfaits du confort : "Le plus important pour une femme c'est l'eau chaude dans la cuisine. C'était très difficile dans les gecekondu "; là-bas «on avait des fourneaux et c'était sale et fatigant». Pourtant, nombre d'entre eux regrettent leur ancienne vie. Certains disent qu'ils seraient restés s'ils avaient pu résister à la pression de leurs voisins, qui voulaient vendre au promoteur le terrain dont leur parcelle faisait partie. Les choses qui leur manquent le plus sont les mêmes : "On laissait les portes grandes ouvertes et les voisins étaient toujours les bienvenus. Ici tu gardes ta porte fermée ; tout le monde vient d'ailleurs, tu ne les connais pas, tu ne leur fais pas confiance." "Là bas, quand on sortait, on tombait sur un voisin. Ici on ne voit jamais personne, tout le monde garde sa porte fermée. " "Dans les gecekondu, quand tu sortais la poubelle, tu voyais une voisine et tu bavardais avec elle, mais quand tu arrives ici tu as tendance à rester à l'intérieur. On s'ennuie tellement... Tu croises des gens dans l'ascenseur et tu les salues par politesse, mais c'est pas des relations chaleureuses." 
Autre avantage souvent cité, la liberté d'action: "Dans le gecekondu, il n'y avait que nous, alors qu'ici il y a trente-trois familles dans l'immeuble et tu dois te conformer à leurs exigences. Ici, on ne peut pas faire ce qu'on veut. Il faut respecter certaines règles quand on vit dans un immeuble à étages". "C'est agréable de vivre dans un gecekondu. Tu peux secouer tes tapis et les laver dans le jardin, ou t'installer dehors pour boire une bonne tasse de thé. Ici tu n'as rien le droit de secouer depuis ton balcon ". Détail intéressant, ici, la majorité des résidents signalent les avantages des deux types d'habitation. Ils évoquent leur gecekondu avec nostalgie, mais il appartient au passé : leur appartement est à présent leur nouveau foyer. Cela tient au fait, me semble-t-il, que la décision de partir leur revient (même s'ils ont parfois subi des pressions). De plus, leur situation est plus positive, ou moins négative, que celle des résidents de cités TOKI ; ils vivent dans des appartements plus grands et de meilleure qualité, tout en n'ayant pas de prêt à rembourser : ils peuvent investir et s'investir dans leur nouveau logement.

\section{- Le gecekondu raconté}

Dépourvus du pouvoir d'influencer le discours dominant contre les gecekondu, leurs habitants peuvent néanmoins, quand l'occasion se présente, raconter l'expérience difficile de leur construction.

La commercialisation, nous l'avons vu, commence dans les années 1980, quand les terrains font leur entrée sur le marché officiel. Les propriétaires de ces terrains passent alors pour "s'enrichir énormément » en échangeant leurs maisons contre des appartements. L'image sociale des gecekondu s'est modifiée en même temps que ceux-ci se transformaient en immeubles d'habitation à plusieurs étages. Ils n'évoquent plus des maisons de pauvres mais le moyen de gagner facilement de l'argent. Les appartements ont été injustement acquis par leurs propriétaires, des "riches sans mérite " [Erman, 2001], un stigmate de plus pour cette population. En outre, dans le cadre des PTU de la TOKI, les propriétaires fonciers de gecekondu sont présentés comme les occupants (isgalci) de terrains qui ne leur appartiennent pas.

Ces stigmatisations suscitent de vives réactions chez les personnes concernées. Au cours des entretiens, les résidents des cités TOKI, mais également des cités privées, m'ont livré le récit des " tourments » endurés pendant la construction de leur maison. Les uns utilisent cette souffrance, que je crois fondée sur une réalité vécue, pour légitimer leur droit au profit qu'ils ont réalisé en négo- ciant leur terrain ; les autres s'en servent pour contester la décision de la токI de ne leur céder qu'un seul appartement en échange de leur terrain, et pour réclamer au gouvernement l'annulation de leur dette.

Nous avons peiné pour bâtir nous-mêmes notre maison. Il est impossible de vous faire comprendre toutes les souffrances que nous avons connues en la construisant. Il n'y avait pas de routes, pas de ligne à haute tension, seulement un chemin pour les vaches. On a transporté les briques et les sacs de ciment sur notre dos. Je me suis retrouvé avec une hernie discale. Si seulement le roc pouvait parler, il vous dirait tout.

En contrepartie de ce dur labeur, ils estiment donc mériter plus que la propriété d'un petit appartement ordinaire qu'ils devront payer des années durant. La valeur d'échange des gecekondu est un sujet d'inquiétude croissant pour leurs propriétaires, tandis que leur valeur d'usage - le mode de vie qu'ils permettaient - occupe une place importante dans les mémoires des populations déplacées.

\section{- Le gecekondu politisé}

Grâce à la mobilisation collective, le quartier de gecekondu acquiert un sens nouveau pour ses habitants, riches de cette nouvelle expérience. Outre les jardins et le sentiment de liberté, sa signification politique est ce qui le rend si précieux, en particulier lorsqu'il devient un espace qui défie le système en place et échappe au contrôle des autorités. Il peut toutefois s'avérer difficile de préserver cet espace et sa signification face à des transformations d'envergure, comme en témoigne Nato Yolu, un quartier alévi ${ }^{15}$ du district ankariote de Mamak. Dans les années 1970, ce quartier est un "territoire libéré » de gauche à l'instar du quartier 1 Mayıs (premier mai), situé dans le district stambouliote d'Ümraniye [Aslan, 2004]. Non seulement ses maisons et ses infrastructures ont été construites collectivement avec l'aide de jeunes gauchistes, mais ses habitants résistent alors aux tentatives d'en faire une décharge municipale, nouant ainsi avec lui des liens très forts. Puis, sous le régime militaire répressif issu du coup d'État de 1980, la vie reprend son cours normal. Les résidents alévis gardent néanmoins cet attachement particulier avec leur quartier, différent des quartiers de gecekondu sunnites, de plus en plus conservateurs et religieux, qui les entourent. Avec le temps, la population du quartier évolue, intégrant de nouveaux venus, mais continue d'élire pendant trois mandats successifs un 
sympathisant de gauche au poste de muhtar. Au début des années 2000, ce chef de quartier élu pour cinq ans mobilise activement la population locale contre la réalisation du projet de transformation urbaine (PTU) par la TOKI, en association avec la municipalité. Il ne parvient pas à sauver le quartier et son mode de vie particulier, mais il réussit à accroître la valeur d'échange du terrain des gecekondu: en écartant la TOKI, les habitants peuvent obtenir plusieurs appartements pour leur parcelle auprès de promoteurs privés, et ainsi réaliser un profit plus important. En conséquence de quoi ce quartier alévi situé à la périphérie d'Ankara, plus mobilisé par une idéologie de gauche que par une identité collective locale, a commencé sa transformation, obéissant à la logique du profit qui règne aujourd'hui dans les villes turques.

La société turque considère les quartiers de gecekondu avec mépris comme des endroits arriérés et ruraux, situés en marge de la vie urbaine. L'opinion prévaut qu'on y vit uniquement par absence de choix. Or, en permettant aux habitants de s'exprimer, mon enquête montre qu'il n'en est rien. Influencés par cette image négative, les habitants de gecekondu eux-mêmes aspirent souvent au confort et au statut social associé à la vie en appartement - avant d'en découvrir les inconvénients. La société méconnaît la valeur qu'ils revêtent pour leurs habitants, en particulier après qu'ils l'ont quitté pour un appartement. Ceux-ci contestent d'ailleurs les propos très durs dirigés récemment contre les gecekondu par le gouvernement néolibéral au pouvoir. Motivé principalement par la perspective d'un profit, l'AKP s'est en effet activement employé à construire une image négative de ces quartiers afin de justifier son intervention.

La logique du profit a ainsi fait son entrée dans l'univers des propriétaires de gecekondu, qui se sont intéressés eux aussi à la valeur d'échange de leur terrain. En particulier lorsqu'ils ne pensaient plus pouvoir rester, ils ont engagé des négociations serrées avec les promoteurs afin d'obtenir un maximum d'appartements en échange de leur parcelle. Cela vaut également pour ceux qui ont tissé des liens étroits avec leur quartier et s'en sont approprié l'espace en le défendant collectivement contre le système, mais aussi en resserrant les rangs de leur groupe minoritaire dans cette partie de la ville. Même s'ils ont essayé de tirer le maximum de leur terrain, seul moyen dont ils disposaient pour améliorer leur situation économique, une majorité d'entre eux regrettent leur vie d'avant. Les acquéreurs d'appartements obtenus auprès de promoteurs privés, de qualité supérieure à ceux de la TOKI, sont assez satisfaits. D'autant que loin de s'endetter ils ont réalisé une plus value. Pourtant eux aussi sont nombreux à regretter leur gecekondu. Les problèmes que ceux-ci posaient ont en effet largement disparu dans cette représentation construite, nimbée de nostalgie. Les relations chaleureuses entre voisins, la solidarité, la proximité de la nature dans les jardins et l'absence des contraintes de la vie en appartement composent ainsi le souvenir d'une existence heureuse.

\section{Traduction de Sylvie Muller [smtrads@orange.fr]}

\section{Notes}

1. En 2004, sous la direction de Jean-François Pérouse, un numéro entier de la revue European Journal of Turkish Studies (Social sciences on Contemporary Turkey) a été consacré au gece$k o n d u$, avec trois articles en français accessibles en ligne (URL : http://ejts.revues.org/45) [Ndt].

2. La токI (Toplu Konut İdaresi), administration du logement collectif (ou HLM turques), dépend directement du Premier ministre. Sous le gouvernement de l'AKP, le pouvoir et les ressources dont elle bénéficie sont importants.

3. La TOKI parle de «Projet de transformation urbaine " (Kentsel Dönüşüm Projeleri). Il s'agit principalement de "Projet de transformation des gecekondu ".

4. Cet article repose sur plusieurs enquêtes de terrain menées à Ankara, puis à Istanbul à des fins comparatives. À Ankara, les recherches ont porté sur plusieurs sites de transformation : la cité de Karacaören-TOKI dans le cadre du projet d'Ankara-Nord et les cités construites par les promoteurs privés du projet du district de Şentepe (2008 et 2012). À Istanbul, les enquêtes concernent la cité de Bezirganbahçe-токI dans le cadre du projet d'Ayazma-Tepeüstü dans le district de Küçükçekmece (2010 et 2011). Elles sont confortées par des recherches antérieures réalisées dans deux quartiers de gecekondu, l'un dans la partie est d'Ankara dans le district de Mamak-Nato Yolu (2000-2003), l'autre dans celui de Çukurca au sud de la ville (1989-1991). Accompagnée d'une assistante, j'ai conduit des entretiens approfondis avec 100 résidents dans le quartier de gecekondu de Nato Yolu, 108 à Çukurca et 60 à Karacaören-TOKI, 25 à Şentepe et 16 à Bezirganbahçe. Nous avons essayé de mettre à l'aise les personnes interrogées afin qu'elles nous fassent part de leurs opinions et de leurs sentiments sur leur existence passée et présente. Je me suis rendue sur place à maintes reprises et j'ai tissé des liens étroits avec plusieurs familles. J'ai pris part à la vie quotidienne et aux événements importants, tant personnels (mariages) que politiques (visite du maire pour le PTU; marche de protestation contre un site de ramassage des ordures près de Nato Yolu). J'ai passé du temps avec ces personnes, j'ai notamment bavardé, mangé parlé au téléphone avec elles, et rendu visite aux voisins. J'ai photographié les sites pour montrer comment les habitants s'intégraient à leur environnement. J'ai également photographié les personnes qui y consentaient, et leur ai ensuite envoyé les clichés en guise de remerciements. Les photos d'enfants furent particulièrement appréciées.

5. Le régime du parti unique prit fin en 1946 avec la création du Parti démocrate, qui gagna les élections de 1950.

6. Même si des familles originaires d'Ankara vivent également dans des gecekondu, moins chers qu'un appartement en ville et plus proches du centre. 
7. L'expression figure entre guillemets pour signifier mes réserves : peut-on vraiment parler de logement social à propos d'appartements vendus à crédit à des pauvres ?

8. Le projet de recherche sur KaracaörenTOKI, subventionné par тUвITAK (The Scientific and Technological Research Council of Turkey), s'intitule "Urban Transformation Projects and the Experiences of Relocation: A Comparative and Longitudinal Approach ».

9. 7000 gecekondu ont été démolis dans le cadre de ce projet.
10. Le projet de rcherche sur Bezirganbahçe-TOKI, subventionné par le Migration Research Program de l'université de Koç, s'intitule "Urban Restructuring, Neoliberal Governmentality and Integration of Migrants : Karacaören-TOKI in Ankara and Bezirganbahçe-TOKI in Istanbul ».

11. La municipalité de Yenimahalle fait de Şentepe un PTU autorisant la construction par des promoteurs privés de tours d'habitation couvrant un pâté de maisons (ada) au lieu des immeubles habituels de quatre étages couvrant une parcelle (parsel) de terrain.
12. Les opposants au régime répressif de l'époque appelaient ainsi une rue, une université ou même un quartier dont ils interdisaient l'accès aux autorités [Ndt].

13. Les groupes qui se forment dans les cités TOKI et leurs relations conflictuelles seront étudiés en détail dans un autre article.

14. L'enquête se déroule environ trois ans après le relogement.

15. Minorité religieuse, islamique de tendance moderniste et républicaine, les alévis constituent 18 à $25 \%$ de la population turque.

\section{Références bibliographiques}

Aslan Şükrü, 2004, 1 Mayıs mahallesi. 1980 Öncesi Toplumsal Mücadeleler ve Kent [Le quartier du premier mai : La mobilisation et la ville avant les années 1980], Istanbul, Iletişim Yayınları.

Bartu-Candan Ayfer et Biray Kolluoğlu, 2008, "Emerging spaces of neoliberalism: A gated town and a public housing project in Istanbul", New Perspectives on Turkey, 39: 5-46.

ERMan Tahire, 1997, "Squatter (gecekondu) housing versus apartment housing: Turkish rural-to-urban migrant residents' perspectives", Habitat International, 28 (1): 91-105.

ERMAN Tahire, 2001, “The politics of gecekondu (squatter) studies in Turkey: The changing representations of rural migrants in the academic discourse", Urban Studies, XXXVIII, 7: 983-1002.

ERMAN Tahire 2011, "Understanding the experiences of politics of urbanization in two gecekondu (squatter) neighborhoods in two urban regimes: Ethnography in the urban periphery of Ankara, Turkey", Urban Anthropology, 40 (1-2): 67-108.

Karaman Ozan, 2008, "Urban pulse - (re)making space for globalization in Istanbul”, Urban Geography, 24 (6) : 518-525.
Keyder Cağlar, 2000, "Liberalization from above and the future of the informal sector: Land, shelter, and informality in the periphery", in Faruk Tabak and Michaeline A. Crichlow (eds.), Informalization: Process and Structure, Baltimore/London, The Johns Hopkins University Press: 119-132.

Kuyucu Tuna et Özlem Ünsal, 2010, "Urban transformation as state-led property transfer: An analysis of two cases of urban renewal in Istanbul", Urban Studies, 47 (7): 1479-1499.

Newman Oscar, 1972, Defensible Space: Crime Prevention Through Urban Design, London, Macmillan.

ŞENYAPIl Tans1, 1982, "Economic change and the gecekondu family", in Ciğdem Kağıtçıbașı (ed.), Sex Roles, Family and Community in Turkey, Bloomington, Indiana University, Turkish Studies 3: 237-248.

Ünsal Özlem et Tuna Kuyucu, 2010, "Challenging the neoliberal urban regime: Regeneration and resistance in Başıbüyük and Tarlabaş1" ", in Deniz Göktürk, Levent Soysal and Ipek Türeli (eds.), Orienting Istanbul: Cultural Capital of Europe ? London/New York, Routledge: 51-70.

YÖNDER Ayşe, 1987, "Informal land and housing markets: the case of Istanbul, Turkey", Journal of the American Planning Association, 53 (2): 213-219.

\section{ABSTRACT}

\section{Ethnography in the Urban Periphery. Understanding the Gecekondu}

This article addresses the perspectives of residents or former residents of gecekondus who, in the processes of urban transformation, have moved to housing estates of high-rise blocks built either by public or private developers. Through the respondents' narratives of the gecekond $u$ as experienced and remembered, emerges the sharp contrast between the positive meanings attributed to the gecekondu by their residents and the negative meanings attributed to it in the hegemonic discourse in society. In the recent practices of the transformation of gecekondu neighborhoods into apartment districts, the exchange value of the gecekondu tends to overshadow its use value. Yet the desire for the gecekondu life preserves its importance for gecekondu people.

Keywords: Housing estate. Gecekondu. Narratives. Rural migrants/urban poor. Turkey. 


\section{ZUSAMMENFASSUNG}

Die Ethnographie des Viertels Gecekondu. Ein selbstgebautes Wohnviertel am Stadtrand.

Der Autor lässt die Einwohner des Viertels Gecekondu zu Wort kommen, die im Rahmen städtebaulicher Maßnahmen in öffentlich oder privat gebaute Wohntürme umgesiedelt wurden. Die Erzählung ihrer gelebten und erinnerten Erfahrung an das alte Viertel steht in krassem Gegensatz zur Abwertung des Viertels in der heutigen Wahrnehmung. Durch die Umgestaltung des Viertes in eine Hochhaussiedlung, scheint der reine Nutzen den Gemeinschaftswert zu verdrängen. Wenngleich die Bewohner des alten Gecedoku alles daran setzen, um seinen ursprünglichenWert zu bewahren.

Stichwörter: Stadtviertel. Gecekondu. Arme ländliche/städtische Migranten. Erzählung. Turquie.

\section{| ÖZET}

\section{Kent Çeperinde Etnografi: Gecekonduyu Gecekondu Halkının Bakışından Anlamak}

Bu makale gecekonduda yaşayan ya da yaşamış olan, ve devlet ya da özel sektör tarafindan inşa edilen çok katlı bloklardan oluşan sitelere taşınan insanların gecekondu hakkındaki görüşlerine odaklanmaktadır. Katılımcıların «deneylenen/ yaşanan gecekondu» ve «hatırlanan gecekondu» hakkındaki anlatıları, gecekonduya yükledikleri olumlu anlamlar ile toplumdaki hakim söylemde gecekonduya yüklenilen olumsuz anlamlar arasındaki keskin zıtlı̆̆ı ortaya koymaktadır. Son zamanlarda gecekondu mahallelerinin apartman semtlerine dönüștürme pratikleri, gecekondunun kullanım değerini değișim değerinin gölgesinde bırakma eğilimini yaratmaktadır. Buna karşın, gecekondu halkı için gecekondu yaşamına duyulan arzu önemini korumaktadır.

Anahtar kelimeler: Gecekondu. Anlatıları. Köylü göçmen/kent yoksulu. Türkiye. 